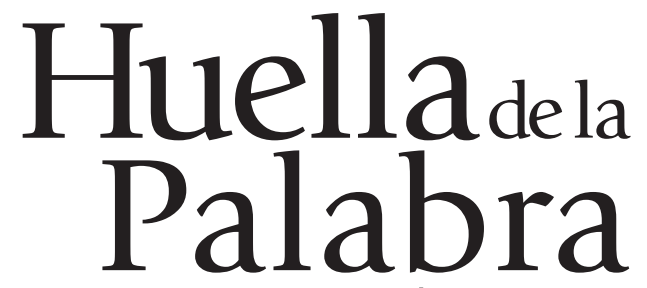

Revista de Investigación Educativa

Universidad La Salle Pachuca

lahuelladelapalabra@lasallep.edu.mx

Teléfono: 01(771) 7170213 ext. 1406

Fax: $01(771) 7170309$

ISSN:En trámite

México

Alma Flores Cabrera

Análisis de un Caso de Depresión

Huella de la Palabra, año 2016, número 10

Universidad La Salle Pachuca

pp. 5-18 



\section{Análisis de un Caso de Depresión}

Alma Flores Cabrera ${ }^{1}$

Resumen

En este trabajo se presenta el desarrollo de intervención desde la psicoterapia sistémica en un caso de depresión. Se irá desglosando detalladamente cada momento de la intervención, desde el análisis de la demanda, los intentos de solución y las hipótesis generadas en cada sesión. Asimismo se detallarán los progresos del tratamiento en cada una de las mismas. Finalmente se realizará una evaluación del proceso terapéutico.

\section{Descripción de la paciente}

H tiene 19 años, es estudiante de segundo cuatrimestre de la carrera en psicología. Su complexión es delgada, tés morena y estatura media. Es puntual en sus sesiones. Su discurso es fluido, aunque con un tono de voz muy bajo en las dos primeras sesiones, en cuanto a su alineo personal siempre acude con ropa deportiva y aseada.

\section{Análisis de la demanda}

La paciente $\mathrm{H}$ realiza una demanda en la que explica que lleva tiempo sintiendo episodios recurrentes de tristeza, en ocasiones con llanto. Durante la primera entrevista se detectan otros motivos latentes como son: peleas recurrentes de los padres, autoestima baja, se siente presionada por toda su familia, y siente rabia e impotencia. Se ayudó a $\mathrm{H}$ a formular su motivo de consulta, ya que en un principio no tenía claro qué era lo que quería tratar, psic. px. "Quisiera logre sentirse más alegre y no forzada, dejar de apartarlo y hacerlo libremente, así como ya no sentir estrés o enojo por una situación que esta fuera de su alcance."

La primera hipótesis en este caso es un Tras-

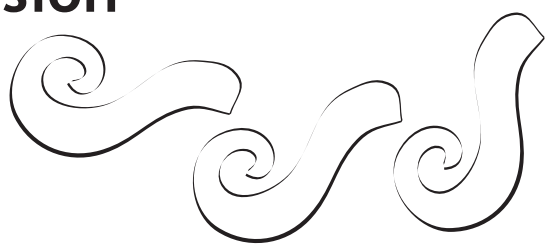

torno del Estado de Ánimo según el DSM-IV (APA, 2005), pudiéndose tratar de un F.32x Trastorno Depresivo Mayor [296.2x] o un F34.1 Trastorno Distímico [300.4]. Poseen características similares, aunque se diferencian por la intensidad de las manifestaciones, la persistencia (crónica o episódica) y el inicio (físico o insidioso). Entre los criterios de diagnóstico, reconocemos en la paciente $\mathrm{H}$ : estado de ánimo de depresión leve que está presente la mayor parte del día, con una duración superior a los dos años, baja autoestima, sentimientos de culpa o tristeza referente a la situación de los padres y el hermano, sentimientos subjetivos de ira excesiva. Ante los datos que tenemos, nos quedarían preguntas abiertas para hacer un diagnóstico certero.

\section{Abordaje del modelo terapéutico}

Históricamente, desde el posmodernismo, se han buscado nuevas maneras de comprender y abordar lo que en otros momentos de la historia se trató como trastornos o enfermedades mentales, y que desde el enfoque sistémico es comprendido como síntoma; es decir, entendido como la forma en que un miembro del sistema hace visible una situación entramada en las dinámicas familiares que de otra manera no podría evidenciarse, entendiendo la singularidad de las experiencias y lo que el sujeto vivencia como único, teniendo en cuenta el contexto y el equilibro entre lo que vivencia el sujeto y su sistemas significativos (Pakman, 2011). Si bien nos construimos en relación con los otros importantes en nuestras vidas, estos tipos de relaciones, estructuras y pautas vienen a influir directamente sobre nuestras configuraciones hereditarias, por ello se hacen

1 Pasante en Psicología. Egresada de la Universidad La Salle Pachuca. 


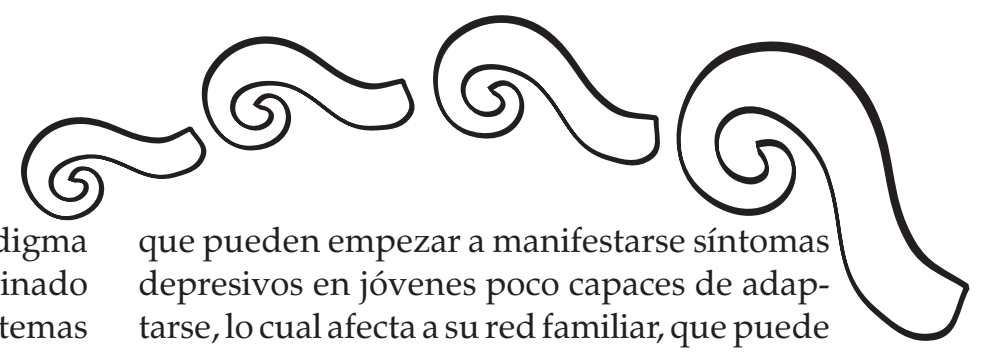

imprescindibles dentro de este paradigma nuevas maneras de abordar un determinado síntoma poniendo en interjuego los sistemas del consultante a nivel familiar, de pares, de pareja y de comunidad.

Para Fruggeri (1996) la psicoterapia es un proceso eficaz cuando el joven cambia sus premisas, narraciones o conversaciones que constituyen el contexto dentro del cual se presentan los síntomas depresivos. En psicoterapia sistémica se trabaja tratando de cambiar la manera de actuar alrededor de la depresión o la manera de verla y las historias que se cuentan acerca de la misma; en este caso nos basaremos en la terapia MRI de Palo Alto con el modelo de terapia centrada en la solución del problema. Se busca tener un mapa de las interacciones sociales que contribuyen tanto a las reacciones depresivas del individuo como a las respuestas que lo alejan de la depresión, así como identificar la pauta de interacción que rodea a la aparición de la depresión para planear alguna intervención que ayude a interrumpir esa pauta de soluciones intentadas, en las cuales se sigue aplicando la misma solución una y otra vez, creyendo que si hacen más de lo mismo lograrán solucionar su problema. Se mantiene presente el cuadro más amplio, los sistemas socioculturales y la manera como las expectativas y creencias de estos contextos tienden a subyugar las vidas de las personas tendiendo a moldearlas dentro de un estereotipo social ideal que, cuando no puede ser alcanzado, contribuye a que la persona se sienta deprimida. Se explora la manera como las personas construyen su depresión: qué hacen, de qué hablan, con quién, qué es lo que piensa y cómo piensan, detectando el impacto de estos aspectos en la aparición e intensidad de la depresión.

Los síntomas depresivos se interpretan como un proceso que se inicia en un acontecimiento que pone en crisis y obliga a un joven a adaptarse y al sistema a reacomodarse proactivamente, lo que pone en marcha factores personales, interpersonales y contextuales. Es así

responder con indiferencia, tolerancia o de modo crítico e impaciente, esto según su estructura específica y sus dinámicas propias. Por ende, las respuestas de los miembros de la familia hacia el joven generalmente mantienen la angustia y los síntomas depresivos, y esto forja un sistema elaborado de retroalimentación que eterniza los síntomas del consultante y afecta los comportamientos y actitudes de los demás. Es importante tener en cuenta que los síntomas depresivos pueden aumentar cuando los familiares prestan una especial atención a los síntomas negativos de la persona, a su vez, los intentos de ayuda o cuidado pueden lograr mantener y afianzar dichos síntomas debido al significado que tienen para el individuo (Linares, 2008).

La terapia sistémica, de acuerdo con Pakman (2011), tiene el propósito de contextualizar los síntomas tanto en el pasado como en el presente de las relaciones del joven con sus relaciones significativas, para ello se toma en cuenta el ámbito interno, familiar, social y cultural, de tal modo que el cambio de uno afecta el cambio del otro, por lo que se devuelve la confianza en las potencialidades de la familia para lograr el cambio en un miembro. Por lo general, la familia consulta cuando cree que el problema se le salió de las manos, y cree que sus herramientas fallaron o son inútiles. En este caso es importante clarificar con el consultante y la familia cuál ha sido el proceso para que se queden ligados a pautas de interacción que perpetúan síntomas depresivos, para luego descubrir nuevos patrones de interacción que devuelvan responsabilidad, confianza y protagonismo a la familia.

\section{Abordaje terapéutico integrador}

Los objetivos de intervención en el caso de la paciente H están fundamentados en la reducción de los síntomas que manifiesta en este caso la depresión. Los cuales serán siempre 


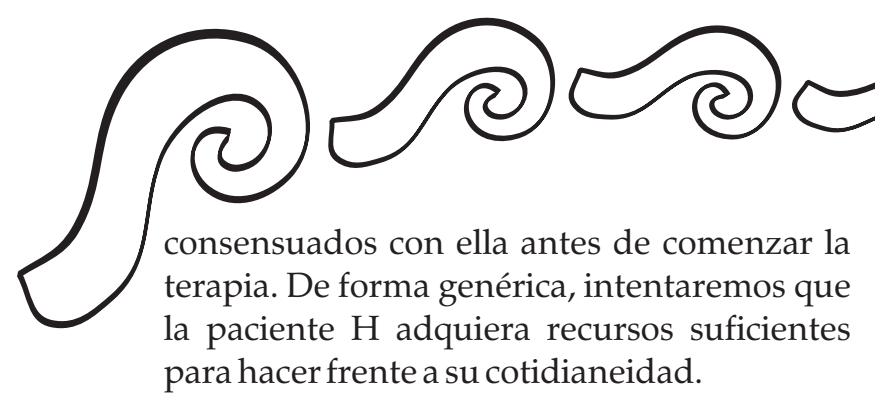

$\mathrm{H}$ tiene que establecer el rol que juega dentro de su familia, tanto con su madre como respecto a la relación de sus padres, así como con su hermano menor.

$\mathrm{H}$ tiene que cambiar la relación de constante apatía que tiene con sus compañeros de la escuela, pues esta forma de interacción supone para ella un foco de frustraciones, angustia $\mathrm{y}$ ansiedad que llevan al mantenimiento de la depresión.

Tiene, también, que eliminar la ambivalencia de sentimientos, ya que no logra identificarlos adecuadamente.

$\mathrm{H}$ tiene que aprender a expresar sus necesidades y sensaciones, y evitar así tener que discutir con su papá y con sus compañeros, etc.

$\mathrm{H}$ tiene que aprender a vivir independientemente, al aceptar lo agradable y lo desagradable, aceptar sus experiencias y motivos por los cuales decide estudiar en Pachuca.

Para conseguir estos objetivos se trabajó mediante el modelo centrado en problemas que, de acuerdo con Fish,Weakland y Segal, se plantean estrategias generales para la solución del problema, proponiendo un cambio en las soluciones intentadas por el paciente (1994); en otras palabras es hacer que el paciente, en este caso $\mathrm{H}$, abandone la solución intentada al proponerle otra respuesta para solucionar su problema, generalmente esta nueva solución tiene que ser exactamente contraria (un giro de $180^{\circ}$ ) a las soluciones intentadas por $\mathrm{H}$.

\section{La terapia de solución de problemas}

En la actualidad existen dos formas de la Terapia de solución de problemas con la finalidad de dar tratamiento a la depresión. La primera está dirigida para aquellos contextos de salud mental especializados (Terapia de solución de problemas sociales), mientras que la otra se ha aplicado en atención primaria (Terapia de solución de problemas para la atención primaria). Las dos están basadas en el modelo propuesto por Nezu y D’Zurilla (1999).

La terapia de solución de problemas sociales es la adaptación original de la terapia de solución de problemas que fue desarrollada por Nezu y Perri (1989). En este modelo, los pacientes participan 8-12 semanas en la Terapia de solución de problemas, tanto a nivel individual como grupal. En la adaptación de la Terapia de solución de problemas se pretende enseñar a los pacientes habilidades de forma secuencial durante 5-6 sesiones. Se aprende algo nuevo en el proceso y en cada sesión, por lo que adquieren gradualmente habilidades; es decir que cada habilidad previa consigue reforzar el dominio de la siguiente habilidad.

Después de 5-6 sesiones, el paciente ha aprendido cómo funciona el modelo y las sesiones siguientes están pensadas para servir de asesoramiento, de modo que el paciente pueda aplicar el enfoque de solución de problemas a los problemas reales mientras se encuentra bajo la dirección del terapeuta. Lo que sigue es una descripción, sesión a sesión, de la Terapia de solución de problemas sociales.

Investigaciones han encontrado que la Terapia de solución de problemas es más eficaz cuando se habla de depresión en niños que las terapias de autocontrol tradicionales (Stark, Reynolds y Kaslow, 1987). De igual forma Salkovskis, Atha y Storer, (1990) mencionan que se han tenido buenos resultados en personas con diagnósticos graves de depresión y con repetidos intentos de suicidio o con frecuentemente pensamientos de ideación suicida.

Es así como la solución de problemas sociales es una técnica de autocontrol que ayuda a la persona a tratar el estrés en muchos de sus casos. Se considera que la solución de proble- 


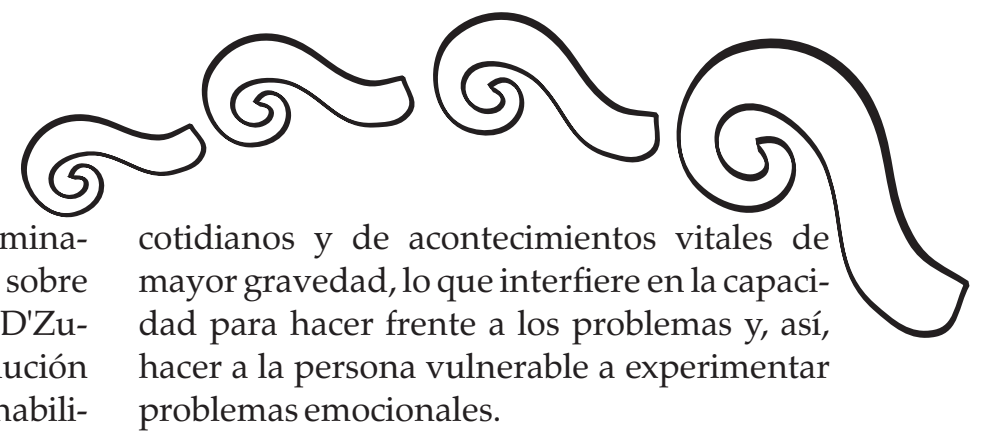

mas es un conjunto de habilidades determinadas que interactúan para tener un efecto sobre la solución rápida y eficaz del problema. D'Zurilla y Nezu (1999) mencionan que la solución de problemas está formada por cinco habilidades. La primera de ellas es la orientación hacia el problema, la cual describe el modo en que uno considera su habilidad para enfrentar un problema. La segunda habilidad consiste en la definición propia del problema, relacionada con la definición concreta y específica del mismo, de esta manera se prosigue al establecimiento de objetivos que se pueden definir y alcanzar. La tercera habilidad se ocupa de la generación de soluciones alternativas, las cuales implican la elaboración creativa de varios métodos para solucionar los problemas y lograr los objetivos propuestos, pero olvidando juicios acerca de la eficacia de las soluciones. En cuanto a la cuarta habilidad, se refiere a la toma de decisiones, que lleva consigo un proceso sistemático para seleccionar la mejor solución a un problema específico. Por último, la quinta habilidad, que consiste en poner en práctica y evaluar la solución elegida, la cual incluye una previa planificación que ha sido puesta en marcha de las soluciones y la evaluación posterior del éxito o fracaso de la solución.

Desde el modelo de solución de problemas, la depresión es consecuencia de la interacción dinámica de los acontecimientos negativos de la vida, los problemas cotidianos, la capacidad de solución de problemas y el malestar emocional de la persona (Nezu, 1986).

En otras palabras los acontecimientos importantes que una persona tiene a lo largo de su vida pueden conducir a problemas cotidianos (la probabilidad de tener una enfermedad). Conjuntamente, los problemas cotidianos pueden desembocar en problemas considerados vitales mucho más serios (discusiones familiares que afectan la relación entre sus miembros). De tal forma, el tener estos problemas puede afectar la capacidad de la persona para afrontar una gran cantidad de problemas

Es por eso que de acuerdo con Nezu y Perri (1989), un método factible para el tratamiento de la depresión consiste en enseñar a la persona deprimida nuevas habilidades de solución de problemas esto al proporcionarle las herramientas necesarias, no solo para resolver los problemas que le están actualmente produciendo la depresión, sino unas que puedan serle útiles en el futuro, y así prevenir las recaídas.

\section{Contacto inicial. Identificación con el tera- peuta y análisis del problema.}

La paciente menciona ser de Oaxaca, actualmente vive en Pachuca sola por motivo de sus estudios. En cuanto a la relación con su papá, comenta que se mantiene alejado tanto de ella como de sus hermanos, mientras que la mamá es sobreprotectora y a todas horas le llama para saber dónde está, y por tal motivo en ocasiones prefiere mentirle sobre dónde está o simplemente no avisarle. En cuanto a su estancia en Pachuca, ella comenta sentirse bien, ya que así no está cerca de los problemas de su familia, como las peleas de sus padres "Psic. Paciente_me siento más liberada".

Por otro lado, mencionó que su papá estuvo enfermo de cáncer hace ocho años y posteriormente su mamá también fue diagnosticada con cáncer de seno; a raíz de esta situación, su hermana mayor se empieza a hacer cargo de ella y de su hermano tomando el rol de la mamá. Respecto a la enfermedad de sus padres, la paciente menciona ser posible candidata a padecerlo, ya que le mencionaron que el cáncer es hereditario en su familia, lo cual le genera una situación de estrés y constante preocupación.

Otro problema por el cual se ve afectada es que 


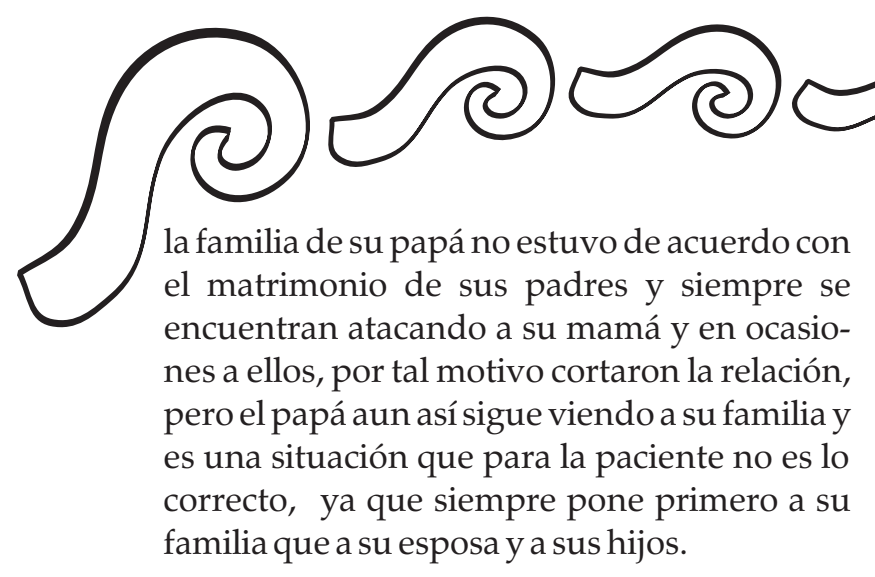

Asimismo, siente una responsabilidad de ser mediadora entre los problemas de sus papás, ya que constantemente la llaman para contarle los problemas que tienen "Psic. Paciente no hay día que no me hablen para decirme algo"; así como con su hermano, a quien le diagnosticaron un ligero retraso, por lo que su mamá insiste en que ella es quien lo puede ayudar por los conocimientos de psicología que tiene, "Psic. Paciente- él no es mi responsabilidad, sí lo quiero y lo voy a ayudar, pero no soy su mamá."

\section{Intentos para enfrentar la situación y resulta-} dos obtenidos.

Como ya se mencionó anteriormente, la paciente encontró su estancia en Pachuca como una salida a sus problemas, aunque en ocasiones se siente igual al no tener a nadie quien la escuche.

Respecto a su hermano, la paciente empezó a alejarse y cumplir el rol solo de hermana y no de mamá, por lo cual el hermano empezó a preguntar si ya no lo quería.

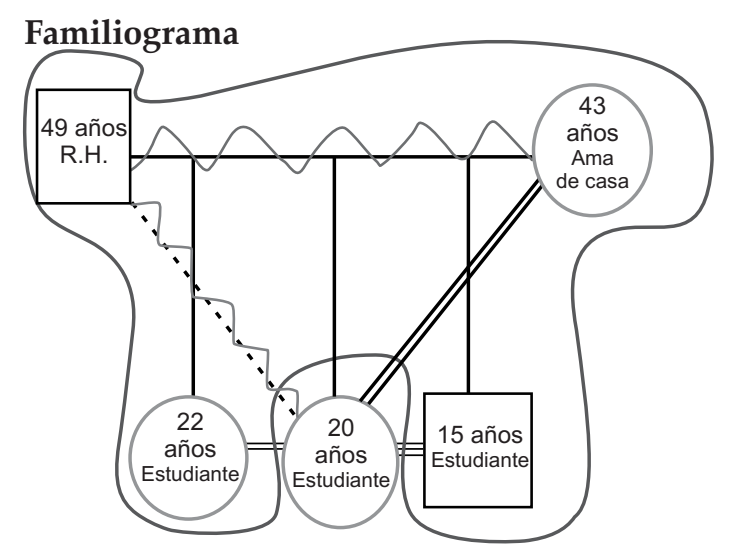

\section{Primera entrevista}

Motivo de consulta: Que la paciente logre sentirse más alegre y no forzada, , así como el que ya no sienta estrés o enojo por una situación que está fuera del su alcance.

Descripción de la organización familiar: El papá es percibido por la paciente como distante, tanto con ella como con sus hermanos y su mamá, además menciona que constantemente se encuentra enojada con él por la indiferencia con la que se muestra hacia su familia, ya que no siente que exista un cariño o que él se preocupe por lo que a ella le pasa.

En cuanto a la mamá, $\mathrm{H}$ menciona que es demasiado sobreprotectora y en ocasiones intrusiva con la paciente, a pesar de la distancia, así como la encargada de reportar todo lo que pasa en Oaxaca mientras la paciente se encuentra estudiando.

La hermana por un tiempo cumplió con el rol de la madre mientras se dio la situación de enfermedad con ambos padres, lo cual para la paciente hizo que madurara; ahora la paciente la percibe como alguien irresponsable hasta con su hija.

Respecto al hermano, la paciente menciona llevar buena relación, es una persona que necesita mucho cuidado y atención debido a que cuenta con un diagnóstico de déficit de atención y retraso mental leve.

La paciente se observa a sí misma como la mediadora de toda la situación familiar, tanto en las peleas de los padres, como en la actitud de la hermana hacia su hija y la presente responsabilidad sobre el cuidado del hermano por cuestión del déficit de atención y el retraso que presenta.

Hipótesis: Existe una depresión leve en la paciente por situaciones de cansancio, tristeza constante y ganas de llorar sin motivo. 


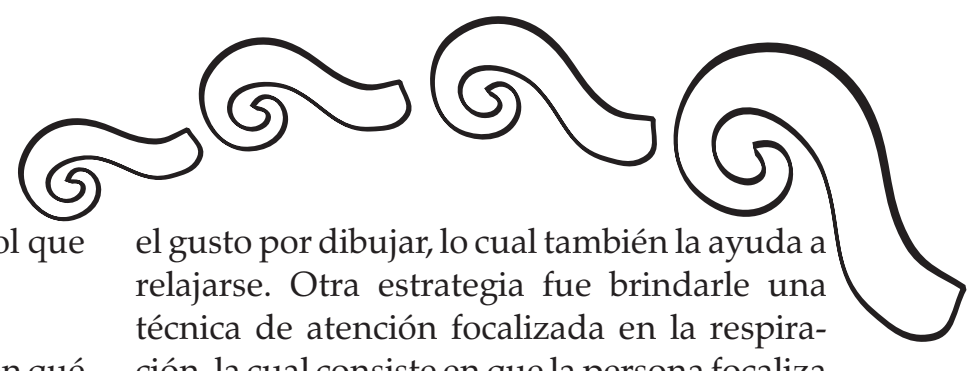

Objetivo terapéuticos: Que identifique en qué momento llega la tristeza, con quién está, dónde está y qué hace cuando se siente triste.

Reflexionar sobre las responsabilidades que tiene $\mathrm{H}$ y si realmente la responsabilidad que toma corresponde a su rol de hija, hermana y estudiante.

\section{Segunda sesión}

Breve resumen de la sesión: mencionó que los momentos de tristeza son más recurrentes por las noches debido al estrés, el cual le provoca cansancio y dolor de cabeza, esto le preocupa porque ha empezado a tomar medicamentos y no quiere generar una dependencia debido a la situación que vivió con la enfermedad de sus dos padres.

Hipótesis: Tanto la tristeza como el enojo que presenta la paciente se origina por no tener un control de la situación, la cual se representa en estrés, y creyéndose ser incapaz de hacer las cosas bien.

El no tener el control de sus emociones le provoca apatía, por tal motivo se presenta la falta de ganas para salir con sus amigos o realizar alguna otra actividad.

Objetivo terapéuticos: Que la paciente logre identificar cuáles son esas situaciones que le generan estrés constante y en qué momento se presenta.

Intervenciones propuestas: Se construyeron estrategias que ayuden a la paciente cuando tenga episodios de enojo y sienta la necesidad de golpear o patalear; asimismo que cuando algo no le salga como ella esperaba, no lo vea como un fracaso sino como una forma nueva de aprendizaje para seguir mejorando Estas estrategias fueron hechas en conjunto con la paciente, ya que durante la sesión mencionó

ción, la cual consiste en que la persona focaliza su atención en su propia respiración, esto permite que el proceso natural e involuntario de la respiración se lleve a cabo bajo total control voluntario, lo que suele generar que la respiración se vaya haciendo cada vez más lenta, más profunda y controlada. Esta técnica es especialmente útil para afrontar situaciones de ansiedad o conflictivas tanto internas como externas.

Que la paciente logre ver cuáles son sus necesidades y no las de su familia, amigos o compañeros, con la finalidad de identificar y responsabilizarse de su propia emoción ante una situación.

Aspectos relevantes significativos: La paciente menciona sentirse más tranquila a comparación de la sesión anterior, y menos deprimida; de esta manera, cuando ella se daba cuenta que se empezaba a sentirse deprimida, buscaba algo en qué ocuparse. También logró identificar el momento de tristeza.

La paciente identifica algunas estrategias que le ayudan a bajar sus niveles de enojo, como el dibujar o salir a correr, lo cual es muy importante, ya que son recursos propios de $\mathrm{H}$, por lo que los podrá seguir haciendo, puesto que son cosas que le gustan y en las que pone interés debido a su gusto por el deporte, en el caso del salir a correr.

\section{Tercera sesión}

Breve resumen de la sesión: En la sesión anterior la paciente mencionó haber podido hablar tranquilamente con su exnovio, lo cual anteriormente le provocaba tristeza al grado de solo con mencionar su nombre se ponía a llorar, debido a la forma en que habían terminado las cosas; ahora mencionó contemplar la situación y hasta que podría volver a darse algo con el exnovio. 


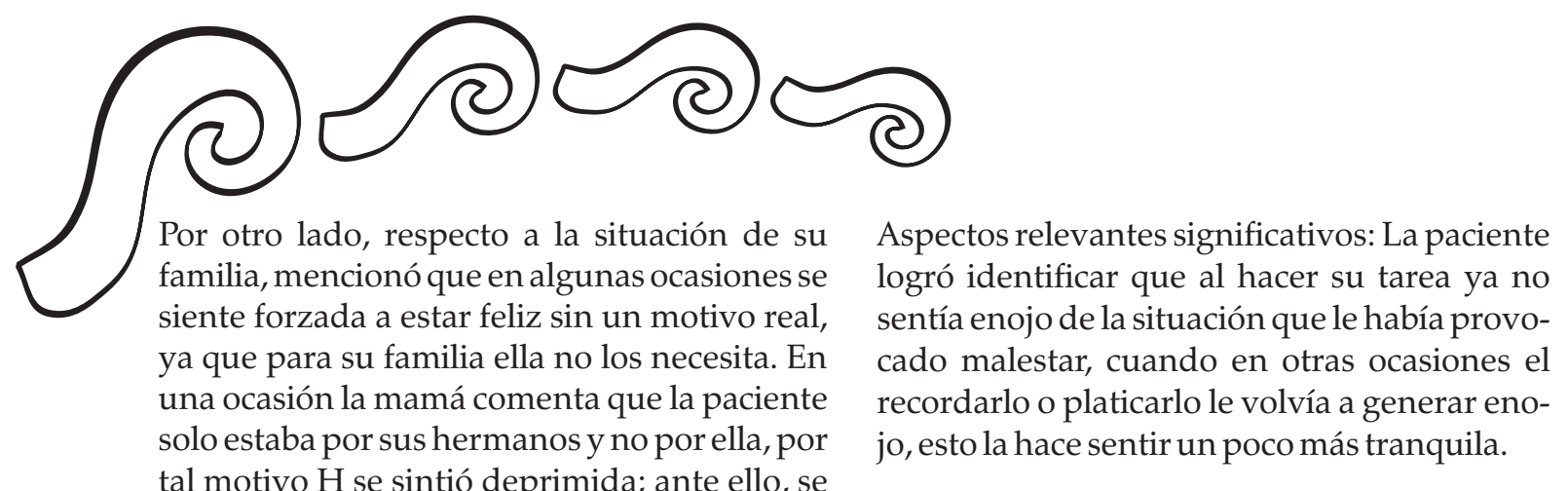
le hizo ver que tal vez estaba malinterpretando el comentario de su madre debido a que quizá ella la cree más fuerte o con mayores capacidades para ser independiente, y como parte del ser independiente la estaban dejando estudiar en otro lugar lejos de casa.

De este modo existen reclamos de parte de su mamá acerca de su estancia en Pachuca, quien le menciona que la abandonó y que ya no la quiere, y que cuando la paciente menciona sentirse estresada, la mamá le hace ver que ella así lo quiso al irse a vivir a otro lugar.

De igual forma, el papá se muestra indiferente a situaciones importantes como cuando la mamá menciona estar enferma, lo cual hace enojar a la paciente.

No existe una verdadera libertad en las acciones y emociones de la paciente, lo cual podría ser el factor que provoca los episodios de tristeza y enojo.

Objetivo terapéuticos: Que la paciente logre establecer una comunicación de diálogo con sus papás y no evadir la situación que le molesta.

Así como identificar situaciones de estrés pensamiento y emoción y posteriormente llegar a una reflexión de la situación.

Intervenciones propuestas: Establecer una comunicación en la cual logre realmente sentirse bien consigo misma y no forzada o teniendo que evadir. Además de identificar si realmente es congruente el malestar que siente con la situación, sin predisponer la respuesta que obtendría; por ejemplo en el caso del papá, quien piensa que no la escuchará porque él nunca lo hace.

\section{Cuarta sesión}

Breve resumen de la sesión: La paciente mencionó sentirse más tranquila después de haber pasado una semana en Oaxaca junto a su familia por motivo del 10 de mayo. Mencionó que cada que escuchaba a sus padres discutir, se alejaba o prefería salir a correr para distraerse, del mismo modo prefería ocuparse en algo para no sentir el enojo de situaciones en las que no tenía el control, ya que anteriormente ella solía pensar cómo solucionar la problemática de sus padres, y qué decirles al respecto.

Por otro lado, mencionó sentir más libertad debido a la confianza que le dieron sus padres al dejarla viajar sola, y anteriormente el viajar con su mamá le generaba estrés, ya que la madre presenta distintas complicaciones de salud, y la paciente durante el viaje se preocupaba por su integridad física. Respecto a las llamadas recurrentes que la mamá realiza, la paciente menciona que han sido reducidas a dos llamadas al día.

Al mismo tiempo mencionó que por un momento su papá le prestó atención al escuchar que estaba acudiendo a un proceso terapéutico, aunque anteriormente ya se lo había comentado. Respecto a la mamá, ya tenía conocimiento de la terapia que recibe la paciente.

La paciente menciona que cuando sus padres se encuentran peleados hay una mejor atención de parte del padre por saber cómo se encuentran o si les hace falta algo, a lo que la paciente refiere que se siente bonito, desafortunadamente no es algo normal dentro de la dinámica familiar.

Hipótesis: Se generó un estado de cambio 


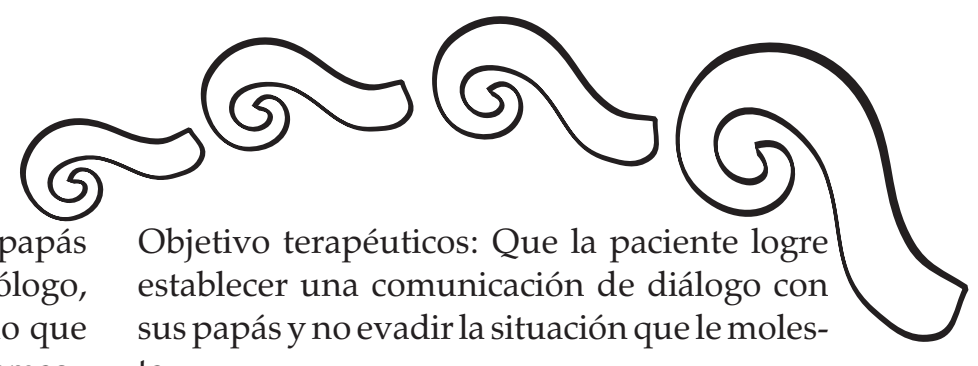

debido a que la paciente comentó a sus papás que se encontraba acudiendo al psicólogo, mediante lo cual obtuvo como resultado que sus padres no la involucren en sus problemas.

Objetivo terapéuticos: Seguir generando una independencia propia y no sujeta a los problemas familiares.

Intervenciones propuestas: Seguir generando nuevas estrategias que permitan controlar los momentos de estrés y de enojo en situaciones que generan conflicto para la paciente.

Aspectos relevantes significativos: Que la paciente lograra identificar la relación que ella promueve al dar y recibir algo de alguna persona, en específico del papá; además que la paciente tomara conciencia de los cambios que ha tenido debido a lo largo de la sesiones.

\section{Quinta sesión}

Breve resumen de la sesión: Se le recordó a la paciente que ésta era la penúltima sesión, también se recopilaron algunos datos que faltaban respecto al familiograma.

La paciente identificó cómo era su comportamiento y su estado emocional respecto a la primera sesión y de qué forma esto ha ido evolucionando hasta el día de hoy, sobre lo cual menciona sentirse mejor y estar más tranquila, con menos carga de estrés en la escuela y con sus padres.

Menciona que ahora se permite el tiempo para distraerse y divertirse y no estar todo el tiempo pensando en los problemas, y gracias a esto las ganas de llorar y los dolores de cabeza han disminuido.

Hipótesis: Se presenta una mejor identificación de emociones por parte de la paciente hacia los momentos en los cuales está bien reflexionar sobre las situaciones problemáticas y aquellos momentos que puede ocupar para divertirse o estudiar.

Así como identificar situaciones de estrés, pensamiento y emoción, y posteriormente llegar a una reflexión de la situación.

Que la paciente establezca más comunicación con sus compañeros.

Intervenciones propuestas: Que establezca comunicación en un momento para reflexionar y que al mismo tiempo le permita desestresarse; asimismo, que esto se convierta en un hábito.

Identificar qué cosas la hacen sentir bien, así como identificar el comportamiento que la paciente tiene respecto a la visita de la mamá y elhermano.

Aspectos relevantes significativos: La paciente logró establecer los momentos que tiene durante el día para realizar las cosas y sentirse mejor consigo misma.

También se logró identificar el rol que juega dentro de su familia y en qué situaciones se puede permitir ayudar en los problemas por los que atraviesan.

La percepción que las personas tenían de ella ha cambiado favorablemente.

\section{Sexta sesión}

Breve resumen de la sesión: Se mencionó a la paciente que ésta sería la última sesión, posteriormente se le comentó la percepción del terapeuta respecto al trabajo de la paciente, así como los cambios que la paciente estuvo logrando dentro del proceso.

Se le plantearon distintas problemáticas para que ella pudiera identificar el cambio y la evolución que estuvo generando; asimismo que 


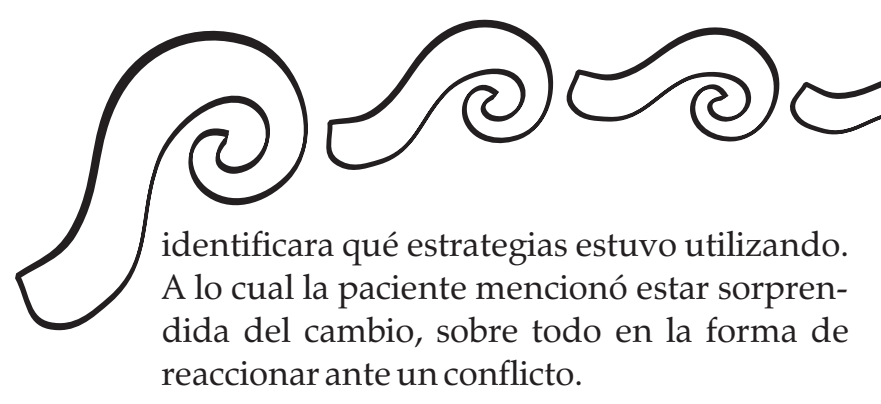

Hipótesis: La paciente cuenta con la habilidad de desarrollar una serie de estrategias que le permitirán enfrentar nuevas situaciones que le generen estrés o malestar emocional.

Objetivo terapéuticos: Que la paciente logre sentirse más libre emocional y conductualmente, y que al mismo tiempo ayude a generar una independencia, la cual le permita hacerse cargo de sus decisiones y emociones.

Que la paciente se diera cuenta que ella misma había hecho el cambio.

Intervenciones propuestas: Se le pide a la paciente que hable con su papá y que le diga que está acudiendo a trabajo terapéutico, para que el papá sienta la necesidad que $\mathrm{H}$ le hable del motivo por el cual decidió tomar terapia, en caso que el padre no mostrase interés en preguntar más allá de lo que $\mathrm{H}$ comentara, no habría problema, pues el objetivo era que ella le comentara cómo se siente.

Aspectos relevantes significativos: Fue importante recalcarle a la paciente el logro de su objetivo, así como el compromiso que tuvo durante el proceso al no llegar tarde y asistir a todas las sesiones.

La paciente $\mathrm{H}$ mencionó que varios de sus amigos empezaban a notar cambios en ella debido a que ya no perdía tan fácilmente el control ni reaccionaba de forma impulsiva cuando algo no le salía como ella quisiera o cuando algo le molestaba.

\section{Evaluación del proceso terapéutico}

1. Metas alcanzadas: La paciente ha logrado identificar cómo bajar sus niveles de le enojo, poniéndose a colorear o a dibujar en ese momento.
Es así como ha generado nuevos recursos que le permiten reaccionar en estas mismas situaciones, como lo es salir a correr o realizar algún deporte.

Que la paciente se sintiera más libre e independiente en cuanto a sus acciones y emociones.

2. Intervenciones propuestas desde el modelo centrado en problemas: Que la paciente identificara la problemática en cuanto a qué situaciones le generan tristeza y estrés, al mismo tiempo la identificación de su rol dentro de la dinámica familiar.

Visualizar qué cosas estaba haciendo la paciente para solucionar la situación referente a la depresión y al estrés, mediante la identificación de situación, conducta y emoción de situaciones de malestar.

Que la paciente logre percibir cambios en su estado de ánimo y una mejoría en la relación con sus padres y sus compañeros de escuela.

3. Aspectos relevantes significativos: La paciente ha mencionado observar cambios significativos cuando está realizando sus tareas, lo cual ha mencionado que le hace sentir bien, menos enojada o triste.

Asimismo, menciona que la relación con sus compañeros ha mejorado y se empieza a involucrar más en actividades de la escuela y fuera de ella; por otro lado, existen comentarios de sus compañeros y amigos que externan que ya no se la ve enojada o que no reacciona agresivamente ante alguna situación.

\section{Conclusiones}

A lo largo de este trabajo, la terapia de solución de problemas es una intervención eficaz y al mismo tiempo flexible que se puede utilizar en diversos contextos y aplicar a distintos problemas; en este caso: la depresión. 
En las tres primeras sesiones la paciente continuaba en un estado sistematizado de la situación, pero no hacía algo por modificar la situación que le genera malestar, simplemente lo evadía.

En la cuarta sesión se percibió un gran cambio en cuanto a la actitud y disposición de la paciente, la cual se mantuvo hasta la última sesión, así como el hecho de anticiparse a las situaciones que le generaban incomodidad, estrés o molestias, por ejemplo, el problema de las peleas entre los padres.

En cuanto al equipo terapéutico, se comentó que la paciente no estaba colaborando con el proceso y se consideró que solo quería ser escuchada, esto durante las primeras tres sesiones. Consideraron necesario confrontar a la paciente para que ella como estudiante de psicología supiera que podía identificar las situaciones de malestar y principalmente las emociones para así generar un cambio.

La intervención con la paciente fue breve y focalizada, en la cual se le enseñó cómo resolver problemas de forma sistemática para que pueda manejar su mundo con mayor eficiencia.

En cuanto a la terapia de solución de problemas en una intervención, es un modelo de ayuda que proporciona una alternativa de tratamiento excelente para aquellos profesionales que tienen que tratar trastornos como la depresión durante un periodo relativamente breve.
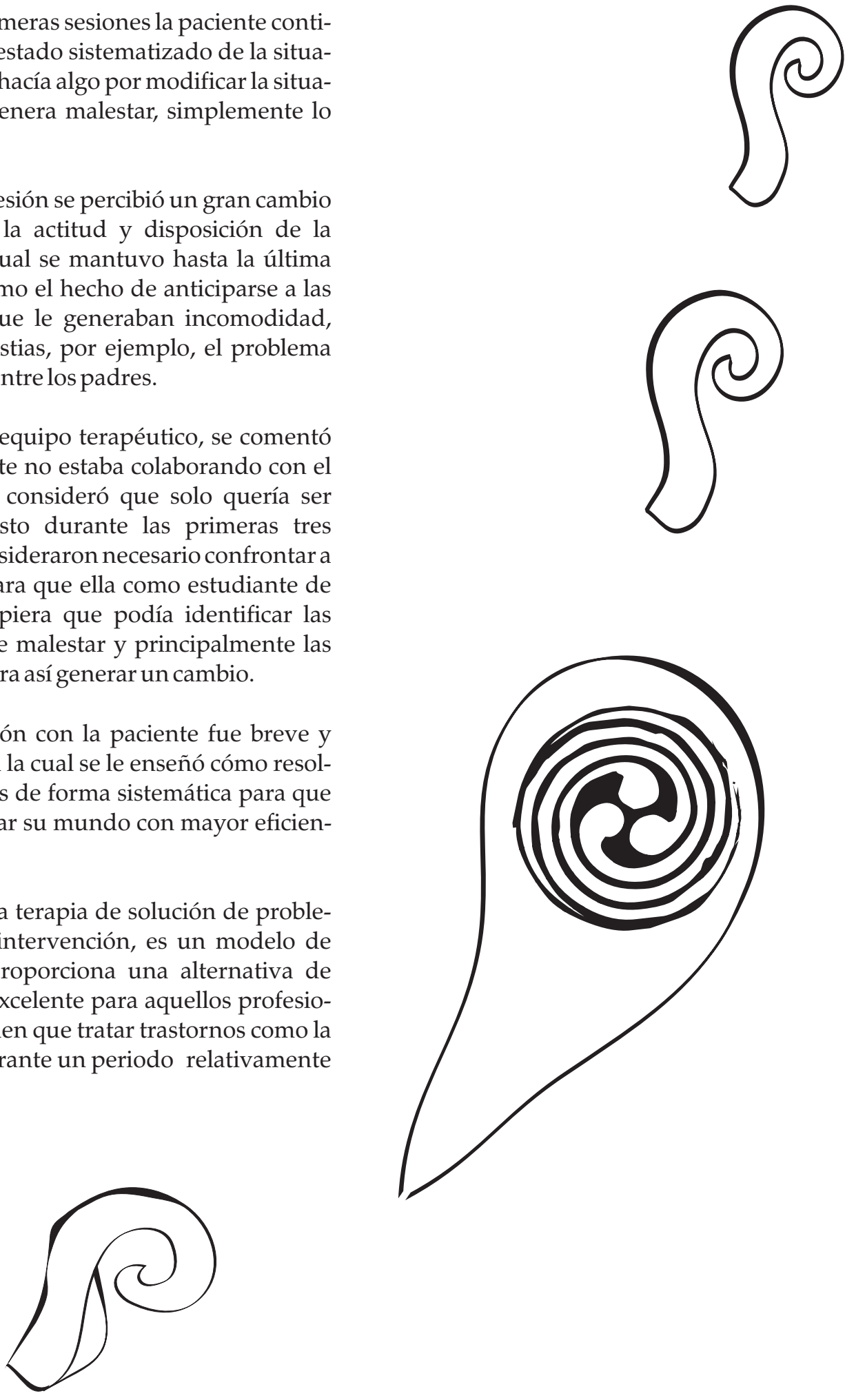


\section{Fuentes de consulta}

American Psychiatric Association (APA). (2005). DMS-IV-TR-AP. Manual diagnóstico y estadístico de los trastornos mentales. Texto revisado. Atención Primaria. Barcelona: Masson.

D'Zurilla, T.J. y Nezu, A.M. (1999). Problem-solving therapy: A social competence approach to clinical intervention. Nueva York: Springer

Fish, R. Weakland, J. Y Segal, L. (1994), La táctica del cambio. Herder. España.

Fruggeri, L., (1996). El proceso terapéutico como construcción social del cambio. En S. McNamme, S. K. J. Gergen. La terapia como construcción social. Barcelona: Paidós.

Linares, Juan L., Campo, C., (2008).Tras la honorable fachada. Los trastornos depresivos desde una mirada relacional. Barcelona: Paidós

Nezu, A.M. (1986). Efficacy of social problem-solving therapy approach for unipolar depression. Journal of Consulting and Clinical Psychology, 54, 196-202.

Nezu, A.M. y Perri M.G. (1989). Social problem-solving therapy for unipolar depression: An initial dismantling investigation. Journal of Consulting and Clinical Psychology, 57, 408- 413.

Pakman, M. (2011). Palabras que permanecen, palabras por venir. Micropolítica y poética en psicoterapia. Barcelona: Gedisa.

Salkovskis, P.M., Atha, C. y Storer, D. (1990). Cognitive-behavioral problem solving in the treatment of patients who repeatedly attempt suicide: A controlled trial. British Journal of Psychiatry, 157, 871-876.

Stark, K.D., Reynolds, W.M. y Kaslow, N.J. (1987). A comparison of the relative efficacy of self-control therapy and a behavioral problem-solving therapy for depression in children. Journal of Abnormal Child Psychology, 15, 91-113.
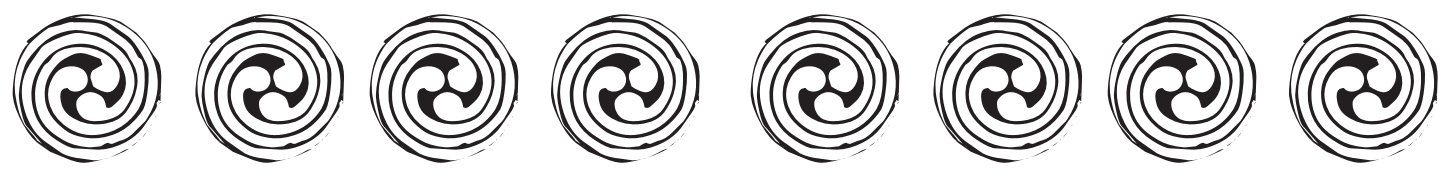

๑) 17 
2018@ 